\section{Bone Tumors: A Practical Guide to Imaging}

\author{
J.S. Wu and M.G. Hochman
}

New York, NY: Springer, 2012, 414 pages, $\$ 89.95$

In the authors' words, the main purposes of this book "are to provide the reader with a practical way to analyze bone tumors and to highlight the most common tumors that one would expect to see in everyday clinical practice." The authors deliberately emphasize the typical appearance and presentation of the tumors they discuss, which, in the opinion of this reviewer, is exactly the right approach. The only practical path to a differential diagnosis of any bone tumor is to cite the tumors that typically have the appearance and occur in the location of the tumor at hand and then admit that once in a while the ultimate pathology will be a surprise.

This book is divided into 10 chapters. The first 3 discuss the basics of an approach to bone tumors, appropriately emphasizing the appearance on conventional radiographs. To anyone familiar with the field, there will be no surprises, and in a book of this type, one would not want surprises! The material is presented in a logical fashion that is easy to understand, with ample use of tables pulling together in list form the main teaching points of the adjacent text.

The next 3 chapters discuss the usual categories of tumor: cartilage tumors, osseous tumors, and fibrous tumors. As is usual in a discussion of a range of tumors, the benign lesions in a particular cell line are treated first, followed by the malignant tumors. Syndromes that produce multiple tumors are explained immediately after the discussion of the relevant solitary lesion. For example, hereditary multiple exostoses are covered immediately after the section on solitary osteochondromas, and Ollier disease and Maffucci syndrome immediately follow the discussion of solitary enchondromas. Recognized subtypes of particular tumors are also discussed. As an example, the section on conventional osteosarcoma is followed by sections on telangiectatic osteosarcoma and parosteal osteosarcoma, as well as brief discussions of high-grade surface osteosarcoma, low-grade intramedullary osteosarcoma, small-cell osteosarcoma, and secondary osteosarcoma. For each discussed tumor, these chapters provide bulletpoint entries on the following topics: synonyms, demographics, origin, location, clinical symptoms, imaging characteristics, malignant potential, treatment, miscellaneous facts, and differential diagnosis. The section on imaging characteristics summarizes the typical appearance on radiographs, CT, MR imaging, and bone scintigraphy including PET and comments on staging workup for malignant tumors.

Chapter 7 is devoted to miscellaneous tumors such as giant cell tumor and Ewing sarcoma. The format is the same as in the chapters on cartilaginous, osseous, and fibrous tumors. Because of the way in which each section is clearly labeled and the sections follow one another in predictable order, this volume has the potential to be a handy reference guide whose ease and speed of use will exceed those of the ubiquitous Internet.

COPYRIGHT (C) 2014 by the Society of Nuclear Medicine and Molecular Imaging, Inc.
Metastases are covered in chapter 8 . For this chapter, the authors wisely depart from the format that had worked so well in previous chapters. The bulletlike discussion of demographics, origin, location, and other topics is used at the beginning of the chapter to describe metastases in general, and there certainly are general rules that can guide evaluation and diagnosis of metastases, no matter their origin. For example, as the authors point out, metastases usually occur in patients over $40 \mathrm{y}$ old and favor the axial skeleton and proximal long bones over the distal extremities. After the general discussion, the authors turn their attention to individual types of metastases that frequently spread to bone. Breast cancer, prostate cancer, lung cancer, renal cell cancer, and thyroid cancer all have separate sections. There is a brief discussion of differentiation of malignant from benign, osteoporotic, or traumatic spinal compression fractures.

Chapter 9 is devoted to lesions that mimic bone tumors, including normal structures, artifacts, and abnormal but nonneoplastic conditions that can look like tumors on imaging. Each is discussed in turn, though in less detail than are the tumors.

The last chapter is devoted to a generous 75 cases on which one can practice differential diagnosis. Just take a piece of paper and cover up the answers!

Without question, the plethora of high-quality illustrations is a winner. Consider, for example, the illustrations on page 172 accompanying the section on aneurysmal bone cysts. Three different examples are given, including an aneurysmal bone cyst in the distal femur, one in the spine, and another in the proximal tibia. The selected images include conventional radiographs, CT scans, and MR images, and there are nice illustrations of the typical fluid-fluid levels on both CT and MR imaging. Arrows and other indicator devices are used judiciously and positioned so they do not cover or distract from the feature they point out. The authors collected most of these illustrations themselves, but they also credit others for certain illustrations, suggesting that they have not hesitated to take advantage of a variety of professional connections to enhance the value of this book.

This book does a fine job of fulfilling its intended role. I really have few complaints. I did find myself curious for more information about the excellent illustrations. I would like to know which ones are pathologically proven (hopefully, the osteosarcomas are and the nonossifying fibromas are not). I would also like to know the age and sex of the patients whose images are used.

This book will be useful to anyone interested in bone tumors, from practicing radiologists to primary care doctors, and particularly to radiology residents.

\section{Tamara Miner Haygood}

University of Texas M.D. Anderson Cancer Center

1515 Holcombe Blvd., Unit 475

Houston, TX 77030

E-mail: tamara.haygood@mdanderson.org 